\title{
Germline mutations in RYR1 are associated with foetal akinesia deformation sequence/lethal multiple pterygium syndrome
}

Arthur B McKie ${ }^{1 \dagger}$, Atif Alsaedii ${ }^{2+}$, Julie Vogt ${ }^{3+}$, Kyra E Stuurman ${ }^{4 \dagger}$, Marjan M Weiss ${ }^{4}$, Hassan Shakeel ${ }^{1}$, Louise Tee ${ }^{2}$, Neil V Morgan ${ }^{2}$, Peter G J Nikkels ${ }^{8}$, Gijs van Haaften ${ }^{6}$, Soo-Mi Park ${ }^{5}$, Jasper J van der Smagt ${ }^{6}$, Marianna Bugiani ${ }^{7}$ and Eamonn R Maher ${ }^{1,2,5,9^{*}}$

\begin{abstract}
Introduction: Foetal akinesia deformation sequence syndrome (FADS) is a genetically heterogeneous disorder characterised by the combination of foetal akinesia and developmental defects which may include pterygia (joint webbing). Traditionally multiple pterygium syndrome (MPS) has been divided into two forms: prenatally lethal (LMPS) and non-lethal Escobar type (EVMPS) types. Interestingly, FADS, LMPS and EVMPS may be allelic e.g. each of these phenotypes may result from mutations in the foetal acetylcholine receptor gamma subunit gene (CHRNG). Many cases of FADS and MPS do not have a mutation in a known FADS/MPS gene and we undertook molecular genetic studies to identify novel causes of these phenotypes.
\end{abstract}

Results: After mapping a novel locus for FADS/LMPS to chromosome 19, we identified a homozygous null mutation in the RYR1 gene in a consanguineous kindred with recurrent LMPS pregnancies. Resequencing of RYR1 in a cohort of 66 unrelated probands with FADS/LMPS/EVMPS (36 with FADS/LMPS and 30 with EVMPS) revealed two additional homozygous mutations (in frame deletions). The overall frequency of RYR1 mutations in probands with FADS/LMPS was $8.3 \%$.

Conclusions: Our findings report, for the first time, a homozygous RYR1 null mutation and expand the range of RYR1-related phenotypes to include early lethal FADS/LMPS. We suggest that RYR1 mutation analysis should be performed in cases of severe FADS/LMPS even in the absence of specific histopathological indicators of RYR1-related disease.

Keywords: Multiple pterygium syndrome, Foetal akinesia, RYR1 mutations, Myopathy

\section{Introduction}

Foetal akinesia deformation sequence syndrome (FADS) is characterised by a variable combination of foetal akinesia, prenatal growth restriction, developmental defects (including cleft palate, cryptorchidism, cystic hygroma, heart abnormalities, intestinal malrotation and lung hypoplasia), arthrogryposis and, in some cases, limb

\footnotetext{
* Correspondence: erm1000@medschl.cam.ac.uk

${ }^{\dagger}$ Equal contributors

'Department of Medical Genetics, University of Cambridge and NIHR Cambridge Biomedical Research Centre, Cambridge Biomedical Campus, Cambridge CB2 OQQ, UK

${ }^{2}$ Centre for Rare Diseases and Personalised Medicine, University of Birmingham, Edgbaston, Birmingham B15 2TT, UK

Full list of author information is available at the end of the article
}

pterygia, so that there is phenotypic overlap between FADS and severe cases of multiple pterygium syndrome (MPS) [1]. Clinically MPS can be divided into the severe lethal form (LMPS) and the milder non-lethal Escobar type (EVMPS). MPS is most commonly inherited as an autosomal recessive trait though autosomal dominant and X-linked cases are described [2-4]. Both MPS and FADS are genetically heterogeneous and although, in some cases, a diagnosis of a specific primary myopathy, metabolic or neurodevelopmental disorder can be made by clinical and pathological investigations, the underlying aetiology is unknown in the majority of cases [5]. Previously, we and others have reported that germline mutations in genes encoding specific components of the 
acetylcholine receptor (AChR) complex at the neuromuscular junction may present with autosomal recessively inherited forms of FADS, LMPS and EVMPS [6,7]. Thus mutations in CHRNG (which encodes the foetal gamma subunit of the acetylcholine receptor) have been associated with FADS, LMPS and EVMPS and mutations in genes that encode other subunits that make up the foetal acetylcholine receptor (CHRNA1, CHRND) or regulators of AChR function (e.g. RAPSN and $D O K 7$ ) have been described in FADS/LMPS [8-10]. Interestingly, mutations in CHRNA1, CHRND, RAPSN and DOK7 can also cause congenital myasthenia syndrome (CMS), a milder disorder that is characterised by muscle fatigability and, rarely, arthrogryposis [11-13].

Identification of the underlying genetic cause of FADS/ MPS facilitates clinical management by providing (a) precise genetic diagnosis, (b) enabling accurate predictions of recurrence risk and prognosis and (c) allowing the possibility of prenatal diagnosis. However, FADS and MPS are genetically heterogeneous and in many cases mutations in acetylcholine receptor-related genes cannot be identified. In order to characterise potential genetic causes of FADS/ MPS in such cases, we undertook molecular genetic investigations in cohorts of FADS, LMPS and EVMPS families that were enriched for autosomal recessively inherited forms of these disorders (i.e. enriched for parental consanguinity) and identified loss of function RYR1 mutations as a cause of early lethal FADS/LMPS.

\section{Material and methods Patients}

66 families with features of FADS/LMPS/EVMPS and no known underlying genetic cause were investigated. In 36 families their clinical phenotype was FADS/LMPS and in 30 the phenotype was EVMPS. Consanguinity was recorded in $48 \%$ of the FADS/LMPS families and $20 \%$ of the EVMPS families. All families gave informed consent, the study was approved by the South Birmingham Research Ethics Committee and performed in accordance with the ethical standards laid down in the 1964 Declaration of Helsinki [14].

\section{Molecular genetic analysis Linkage analysis}

A genome-wide linkage scan was carried out using the Affymetrix $250 \mathrm{~K}$ Human SNP Array 5.0 on DNA from stored foetal material of two affected siblings from a consanguineous family affected with FADS/LMPS. This scan excluded linkage to known FADS/LMPS genes and an $\sim 10 \mathrm{Mb}$ prime candidate region on chromosome 19 was identified and further evaluated by typing the parents and DNA from three affected foetuses with microsatellite markers (details on request and see Figure 1A).

\section{RYR1 sequencing}

$R Y R 1$ gene sequencing was performed after amplification of all 106 coding exons. Initially, sequencing was performed on whole genome amplified DNA (Qiagen REPLI-g kits) and candidate variants were then confirmed on stock DNA samples. Flanking primers were designed from genomic sequence 20-80 nucleotides upstream or downstream from encoding exons. PCR products were sequenced in forward and reverse orientations using standard BigDye $^{\mathrm{R}}$ Terminator v3.1 cycle sequencing. Details of primer sequences are available on request. Sequence traces from each of the DNAs analysed was compared to the reference sequence from the ENSEMBL database (GRCh37: CM000681.1 - NM_000540; transcript ENST00000355481). The segregation of sequence variants was checked in other family members (when available) by BigDye ${ }^{\mathrm{R}}$ Terminator v3.1 sequencing. Frequency information for RYR1 variants was sought from the NHLBI Exome variant server [http://evs.gs.washington.edu/EVS/] if available and the prediction of possible effects of any amino acid substitution was achieved with the PolyPhen-2 tool [http://genetics.bwh.harvard.edu/pph2/].

\section{Histopathological analysis}

Histopathological analysis was performed on tissue obtained at autopsy from two fetuses of family MPS001 (12+ 6 and $14+0$ weeks GA, respectively) and two age-matched controls $(13+0$ and $13+4$ weeks GA, respectively) retrieved from the autopsy archive of the VU University Medical Center, Amsterdam, The Netherlands. sixum thick formalin-fixed paraffin-embedded tissue sections were processed according to standard protocols [15]. Histochemical staining included Hematoxylin \& Eosin, Gomori trichrome and alizarin red $\mathrm{S}$ for calcium. After heat-induced antigen retrieval in $0.01 \mathrm{M}$ citrate buffer (pH6), immunohistochemical staining was performed with antibodies against desmin (Abcam, 1:500), myosin heavy chain slow (Abcam, 1:100), active caspase 3 (Dako, 1:500), CD3 (Dako, 1:250), CD20 (Dako, 1:50) and CD45 (Dako, 1:100). Immunoreactivity was detected with 3,3'-diaminobenzidine as chromogen. Tissue sections were photographed using a Leica DM6000B microscope (Leica Microsystems). Omitting primary antibodies yielded no significant staining.

Ultrastructural analysis was performed on muscle tissue retrieved from formalin fixed material. The tissue was deparaffinised in xylene $\left(60\right.$ minutes at $\left.70^{\circ} \mathrm{C}\right)$, rehydrated, fixed with $2 \%$ glutaraldehyde in $0.1 \mathrm{M}$ sodium cacodylate buffer ( $\mathrm{pH} 7.4$ ), post-fixed in $2 \%$ osmium tetroxide and embedded in epoxy resin. Ultrathin sections were stained with uranyl acetate and lead citrate and viewed in a FEI Technai 12 electron microscope.

The pictures were acquired as TIFF files and images were optimized for brightness and contrast using Photoshop, version 7.0 (Adobe systems, San Jose, CA). 


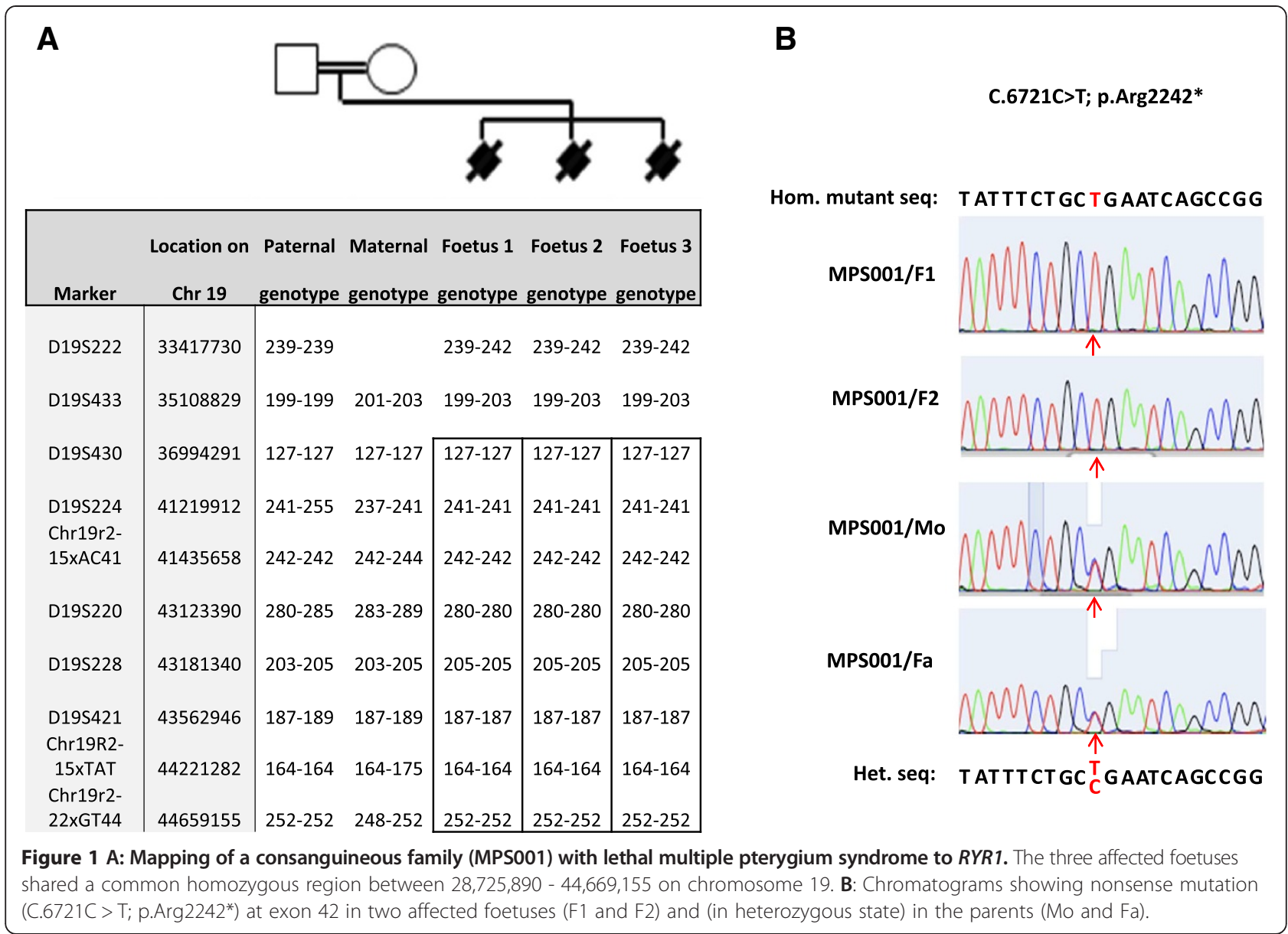

\section{Results}

\section{Clinical and molecular genetic analysis in MPS001 family}

Genome wide linkage analysis was performed on a consanguineous family from The Netherlands (see Family MPS001 in Table 1) that had had six pregnancies affected by FADS/MPS. The proband (F2) presented was the second pregnancy that was terminated at $12+6$ weeks of gestation because of an increased nuchal translucency of $9 \mathrm{~mm}$, fetal akinesia and joint contractures. There was no intrauterine growth restriction. At post mortem examination there was a cystic hygroma, lung hypoplasia, webbing of both elbows and knees and arthrogryposis. The calvaria were absent due to birth trauma however there were no skeletal abnormalities (histopathology results are described below). In the family history, the first pregnancy was terminated because of increased nuchal translucency of $12 \mathrm{~mm}$, fetal akinesia and a unilateral club foot. Additionally, the third, fourth, sixth and seventh pregnancy were terminated, because of increased nuchal translucency and fetal akinesia. The fifth pregnancy ended in an early miscarriage.

After genotyping of DNA from two affected foetuses (first and second foetuses) by high resolution Affymetrix SNP arrays (Genome-Wide Human SNP Array 5.0) a large homozygous region on chromosome 19 was selected for further analysis. Genotyping with microsatellite markers (see Table 2), was then performed on parental DNA samples and DNA from three affected pregnancies. The additional genotyping defined the candidate autozygous region as Chr19; 35108829-44484993) (see Figure 1A). A microsatellite marker (D8S373) that mapped within the only other candidate autozygous region $>2 \mathrm{Mb}$ was demonstrated to be heterozygous in two affected foetuses and so excluded linkage to the chromosome 8 candidate interval (data not shown). At the time of analysis the candidate region on chromosome 19 contained 345 known or predicted genes including the ryanodine receptor 1 (skeletal) (RYR1) gene (Chr19;38924340-39078204).

\section{RYR1 mutation analysis}

Sequencing of RYR1 in an affected foetus (F2) from Family MPS001 revealed a homozygous $R Y R 1$ nonsense mutation (NM_000540.2(RYR1): c.6721C > T; p.(Arg2241*)). Further analysis demonstrated that a second affected foetus (F1) was also homozygous for c.6721C > T, whereas both parents were heterozygous for the nonsense mutation (see Figure 1B). Subsequently DNA from the F3, F4, F6 and F7 were also shown to be homozygous for the mutation 
Table 1 Clinical features of 66 probands in which RYR1 mutation analysis was performed

\begin{tabular}{|c|c|c|c|}
\hline Family number & Phenotype & Ethnicity & Consang. \\
\hline MPS001 & LMPS & White & $Y$ \\
\hline MPS002 & LMPS & South Asian & Y \\
\hline MPS003 & LMPS & Middle Eastern & Y \\
\hline MPSO04 & LMPS & South Asian & Y \\
\hline MPS005 & LMPS & South Asian & Y \\
\hline MPS006 & LMPS & South Asian & Y \\
\hline MPS007 & LMPS & Not available & $\mathrm{N}$ \\
\hline MPS008 & LMPS & White & $\mathrm{N}$ \\
\hline MPS009 & LMPS & White & $\mathrm{N}$ \\
\hline MPS010 & FADS & Middle Eastern & Y \\
\hline MPS011 & LMPS & Not recorded & Y \\
\hline MPS012 & LMPS & North African & Y \\
\hline MPS013 & LMPS & White & $\mathrm{N}$ \\
\hline MPS014 & LMPS & South Asian & Y \\
\hline MPS015 & LMPS & White & $\mathrm{N}$ \\
\hline MPS016 & LMPS & Not available & Y \\
\hline MPS017 & LMPS & North African & Y \\
\hline MPS018 & FADS & White & $\mathrm{N}$ \\
\hline MPS019 & LMPS & White & N \\
\hline MPS020 & LMPS & White & $\mathrm{N}$ \\
\hline MPS021 & LMPS & South Asian & Y \\
\hline MPS022 & FADS & Middle Eastern & Y \\
\hline MPS023 & LMPS & Middle Eastern & Y \\
\hline MPS024 & LMPS & White & $\mathrm{N}$ \\
\hline MPS025 & FADS & Mixed race & $\mathrm{N}$ \\
\hline MPS026 & FADS & White & $\mathrm{N}$ \\
\hline MPS027 & LMPS & Not available & Y \\
\hline MPS028 & LMPS & Not available & $\mathrm{N}$ \\
\hline MPS029 & FADS/LMPS & Not available & $\mathrm{N}$ \\
\hline MPS030 & FADS/LMPS & Not available & $\mathrm{N}$ \\
\hline MPS031 & LMPS & White & $\mathrm{N}$ \\
\hline MPS032 & LMPS & White & $\mathrm{N}$ \\
\hline MPS033 & LMPS & White & $\mathrm{N}$ \\
\hline MPS034 & EVMPS & Not available & Y \\
\hline MPS035 & EVMPS & South Asian & Y \\
\hline MPS036 & EVMPS & White & $\mathrm{N}$ \\
\hline MPS037 & EVMPS & Not available & $\mathrm{N}$ \\
\hline MPS038 & EVMPS & White & $\mathrm{N}$ \\
\hline MPS039 & EVMPS & White & $\mathrm{N}$ \\
\hline MPS040 & EVMPS & White & $\mathrm{N}$ \\
\hline MPS041 & EVMPS & White & N \\
\hline MPS042 & EVMPS & White & $\mathrm{N}$ \\
\hline MPS043 & EVMPS & South Asian & $\mathrm{N}$ \\
\hline
\end{tabular}

Table 1 Clinical features of 66 probands in which RYR1 mutation analysis was performed (Continued)

\begin{tabular}{|c|c|c|}
\hline MPS044 & EVMPS & South American \\
\hline MPS045 & EVMPS & South Asian \\
\hline MPS046 & EVMPS & South Asian \\
\hline MPS047 & EVMPS & White \\
\hline MPS048 & EVMPS & African \\
\hline MPS049 & EVMPS & Not available \\
\hline MPS050 & EVMPS & White \\
\hline MPS051 & EVMPS & White \\
\hline MPS052 & EVMPS & White \\
\hline MPS053 & EVMPS & White \\
\hline MPS054 & EVMPS & White \\
\hline MPS055 & EVMPS & White \\
\hline MPS056 & EVMPS & White \\
\hline MPS057 & EVMPS & South American \\
\hline MPS058 & EVMPS & White \\
\hline MPS059 & EVMPS & African \\
\hline MPS060 & EVMPS & White \\
\hline MPS061 & EVMPS & White \\
\hline MPS062 & EVMPS & White \\
\hline MPS063 & EVMPS & White \\
\hline MPS064 & LMPS & Middle Eastern \\
\hline MPS065 & LMPS & White \\
\hline MPS066 & LMPS & North African \\
\hline
\end{tabular}

Clinical phenotype (FADS = Foetal Akinesia Deformation Sequence, LMPS = Lethal Multiple Pterygium Syndrome; EVMPS = Escobar Variant Multiple Pterygium Syndrome), ethnic origin and presence of parental consanguinity is recorded).

(data not shown). The RYR1 c.6721C > T mutation was previously detected (rs200563280), in the heterozygous state in 1 of 6503 individuals genotypes listed in the exome variant server (http://evs.gs.washington.edu/EVS) (no homozygous genotypes were detected at an average read depth of 49). In order to assess the potential role of recessive RYR1 mutations in FADS, and/or MPS we proceeded to screen a further 66 probands for germline RYR1 variants. Two further potential candidate homozygous mutations were detected, a novel in-frame deletion of 27 nucleotides (NM_000540.2(RYR1): c.2097_2123del p.(Glu699_Gly707del)) and an in-frame deletion of 3 nucleotides c.7043delGAG p.(Glu2347del) (rs121918596)) (see Table 3). Each of the candidate mutations were detected in the homozygous state in individuals with FADS/MPS.

Clinical and molecular genetic analysis in MPS002 family A novel in-frame deletion of 27 nucleotides (NM_000540.2 (RYR1):c.2097_2123del p.(Glu699_Gly707del)) was detected in Family MPS002 of Pakistani origin (Figure 2A).

The family presented at eighteen weeks of pregnancy with a female foetus with bilateral talipes and fixed flexion 
Table 2 Microsatellite markers employed in Mapping of chromosome 19 candidate region in consanguineous family (MPS001)

\begin{tabular}{|c|c|c|}
\hline Marker & Genomic position & Source \\
\hline D19S222 & Chr.19-28,725,890-28,726,217 bp & http://rgd.mcw.edu/rgdweb/report/marker/main.html?id=1341900 \\
\hline D19S433 & Doesn't map to Chr.19 assembly & http://rgd.mcw.edu/rgdweb/report/marker/main.html?id=1298168 \\
\hline D19S430 & Chr.19-32,302,451-32,302,741 bp & http://rgd.mcw.edu/rgdweb/report/marker/main.html?id=1657125 \\
\hline D19S224 & Chr.19-35,493,932-35,494,196 bp & http://rgd.mcw.edu/rgdweb/report/marker/main.html?id=1336138 \\
\hline Chr19r2-15xAC41 & Chr.19-41,435,658-43,123,394 & ftp://ftp.broad.mit.edu/pub/human_STS_releases/ \\
\hline D19S220 & CHR.19-34,8798,595-34,879,871 BP & http://rgd.mcw.edu/rgdweb/search/markers.html?term=D19S220\&speciesType=1 \\
\hline D19S228 & Chr.19-34,937,645-34,937,798 bp & http://rgd.mcw.edu/rgdweb/report/marker/main.html?id=1338234 \\
\hline D19S421 & Chr.19-38,871,106-38,871,460 bp & http://rgd.mcw.edu/rgdweb/report/marker/main.html?id=1337182 \\
\hline Chr19R2-15xTAT & Chr.19-44,221,282-44,669,155 & ftp://ftp.broad.mit.edu/pub/human_STS_releases/ \\
\hline Chr19r2-22xGT44 & Chr.19-44,221,282-44,669,155 & ftp://ftp.broad.mit.edu/pub/human_STS_releases/ \\
\hline
\end{tabular}

of the elbows. There was evidence of fetal hydrops on the ultrasound scan including a large cystic hygroma, subcutaneous oedema, ascites also pleural and pericardial effusions. A cardiac ventriculoseptal defect was also suspected. The pregnancy was terminated at 19 weeks of gestation. Post mortem examination revealed no intrauterine growth restriction, but the foetus was considered to be dysmorphic (protuberant eyes, hypertelorism, a flat nose, low set ears), with a complete cleft palate. There was fixed flexion of all the limb joints and a fracture of the proximal left humerus. Pterygia were present between the inferior margin of the mandible and the anterior chest wall and across the elbows. The heart was normal. There were no other congenital abnormalities noted. The brain was structurally and histologically normal. Muscle histopathological analysis is described below.

This candidate RYR1 c.2097_2123del mutation was present in a homozygous state in two affected foetuses and was found to be heterozygous in both parents. The in-frame deletion was predicted to result in a missense substitution (p.(Glu699Asp)) followed by a deletion of 9 amino acids (GlyTrpGlyGlyAsnGlyValGlyAsp) from the SPRY2 domain of the RYR1 gene product. All 9 of these deleted amino acids were conserved in vertebrate $R Y R 1$ orthologues including zebrafish and 6 of 9 amino acids were conserved in C.elegans (see Figure 2A).

Clinical and molecular genetic analysis in MPS003 family In a consanguineous family (MPS003) with LMPS, a homozygous in-frame deletion (NM_000540.2(RYR1):
c.7043delGAG, p.(Glu2347del) (rs121918596)) was identified in exon 45 in the proband (Figure 2B). This female foetus was the third pregnancy of a consanguineous Palestinian couple. In the pregnancy there was a suggestion of polyhydramnios. On ultrasound examination there were reduced foetal movements and joint contractures were present. The foetus had a cystic hygroma, a hydrothorax, a short neck, a kyphosis and a short trunk due to a scoliosis. The pregnancy was terminated at $23+3$ weeks of gestation. On examination there was no evidence of intrauterine growth restriction. The foetus had craniofacial anomalies including downslanting palpebral fissures, hypertelorism, a broad nasal bridge, a small mouth and high arched palate, low set ears, a short broad neck and a scoliosis. There were flexion contractures of the shoulders, elbows, wrists, hips, knees and ankles. There were clenched hands but no finger contractures. There was webbing of the axillae, elbows, hips, knees and ankles, and rocker bottom feet. There was a severe scoliosis but no bony abnormalities identified on the radiograph. There was pulmonary hypoplasia. There were no abnormalities of the brain or spinal cord. The muscle in the extremities showed increased fibre size disproportion on microscopy (Figure 3). Cardiac muscle histology was normal.

The karyotype was normal. CHRNG, CHRNA1, CHRNB1, CHRND, RAPSN and DOK7 analysis was normal. Subsequently a fourth pregnancy of a male foetus was terminated at $21+3$ weeks of pregnancy because of evidence of foetal akinesia. On examination of the foetus no growth restriction was noted. There was a cystic

Table 3 Rare RYR1 variants detected in families with FADS/LMPS phenotypes

\begin{tabular}{llll}
\hline Family ID & DNA change & Protein change & Genotype \\
\hline MPS001 & c.6721C $>T$ & p.Arg2241 STOP & Homozygous \\
MPS002 & c.2097_2123del & p.(Glu699_Gly707del) & Homozygous \\
MPS003 & c.7043delGAG & p.(Glu2347del)) & Yes \\
\hline
\end{tabular}




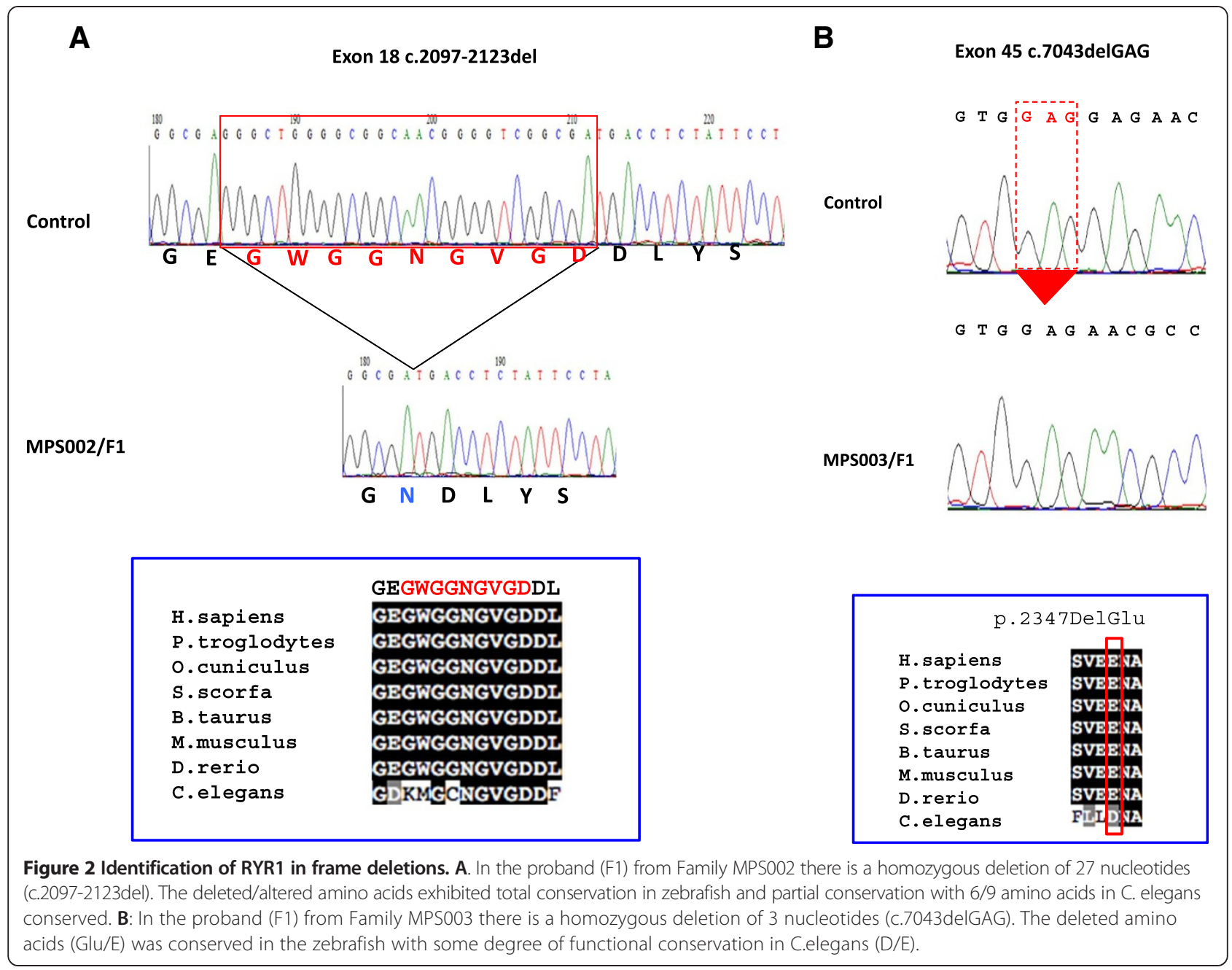

hygroma. The foetus had downslanting palpebral fissures, a broad nasal bridge, a small mouth with a high arched palate. There were contractures of the large and small joints with webbing of the axillae, elbows, hips and knees with pronounced kyphosis, 11 pairs of ribs, but no other bony abnormalities on radiography.

The foetus had lung hypoplasia and there was a small atrial septal defect. There were no abnormalities of the brain or spinal cord. Skeletal muscle microscopy showed increased fibre disproportion. Immunohistology of the structural proteins (alpha-actin, dystrophin 1,2 and 3, alpha, beta, delta, and gamma sarcoglycan, alpha and beta dystroglycan, dysferlin, caveolin-3, merosin laminin alpha-2 chain, merosin M-chain, myosin heavy chain and spectrin-1) did not identify the pathogenesis of the myopathy (data not shown). It was concluded that the foetus had a non specific myopathy. The couple had one miscarriage and they had an older daughter with a severe undiagnosed metabolic disorder. There were no instances of malignant hyperthermia in the family and the mother had general anaesthesia on several occasions without any problems. The RYR1 c.7043delGAG candidate mutation was present in both parents in the heterozygous state and there were no other relatives available for analysis. The c.7043delGAG deletion is predicted to result in loss of a glutamic acid residue at codon 2347 in the MHS/CCD hotspot region 2 of the $R Y R 1$ gene product (Figure 4). This residue is conserved in zebrafish and although the amino acid sequence around this residue is divergent in C. elegans, the glutamic acid is conserved. Polymorphic variation at codon 2347 was not present in $>13,000$ RYR1 alleles reported on the exome variant server (http://evs.gs. washington.edu/EVS), but the c.7043delGAG deletion was previously described, in the heterozygous state, in affected members (total 5 cases) of two unrelated families that presented with malignant hyperthermia [16].

\section{Histopathological studies Family MPS001}

Histopathological analysis was performed on intercostal skeletal muscle from the two foetuses of family MPS001 and two age-matched controls, as this was the only 


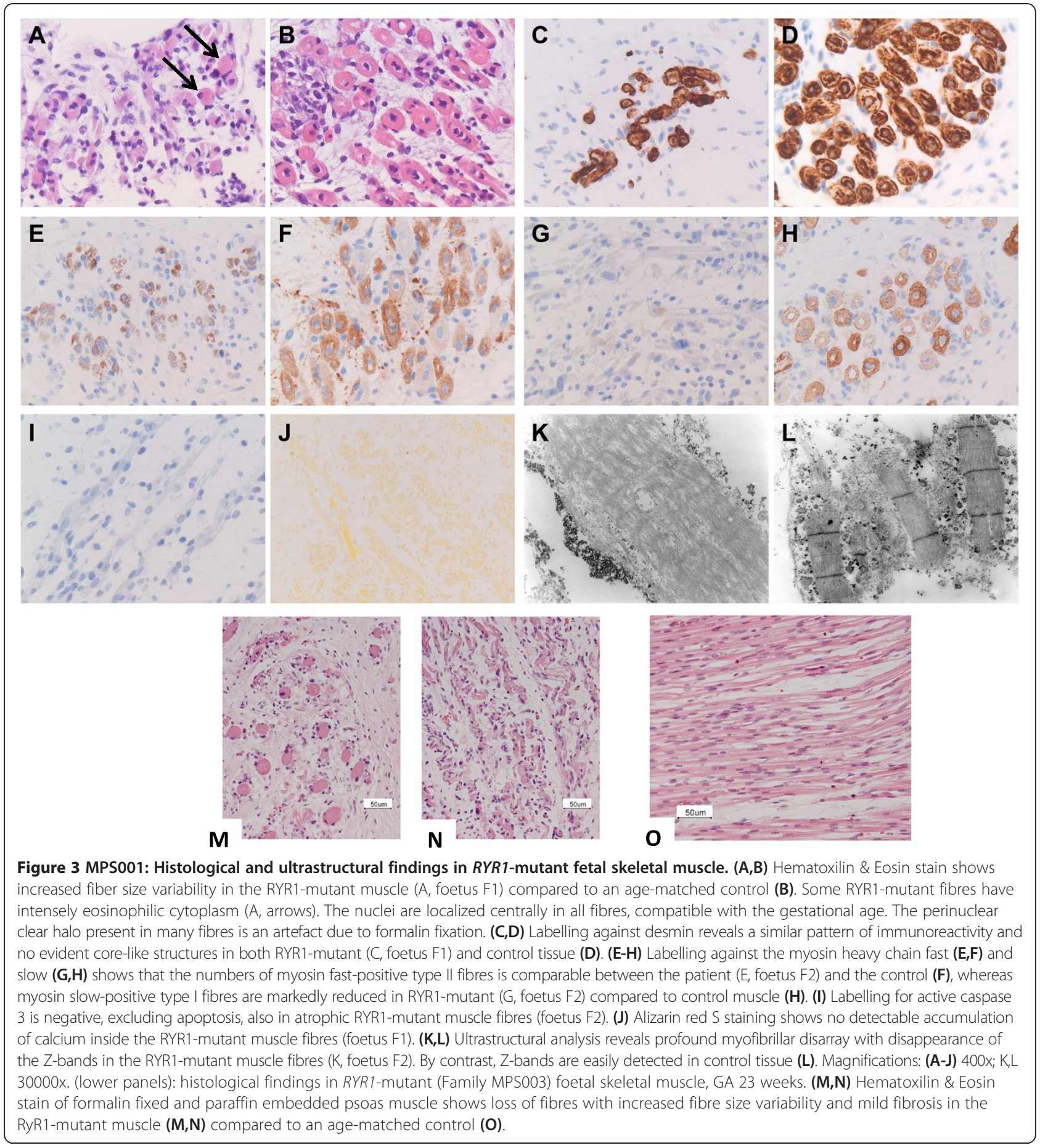

tissue available for both patients. Quantitative histological examination showed marked structural abnormalities in the RYR1-mutant skeletal muscle, including fibre loss, increased fibre size variability and increased endomysial spacing with fibrosis (Figure 3A,B). In agreement with the gestational age, muscle fibres of both patients and controls contained centrally positioned nuclei. Sparse muscle fibres with intense eosinophilic cytoplasm were detected in the patients, but not in the controls (Figure 3A,B) which was not due to calcium accumulation (Figure 3J). Staining against desmin, an intermediate filament protein typically expressed in muscle tissue, revealed no core-like structures (Figure 3C,D). Fibre type distribution, assessed by staining against the slow and fast myosin heavy chain, showed a marked reduction of myosin slow immunoreactivity, suggesting preferential hypotrophy of type I fibres 


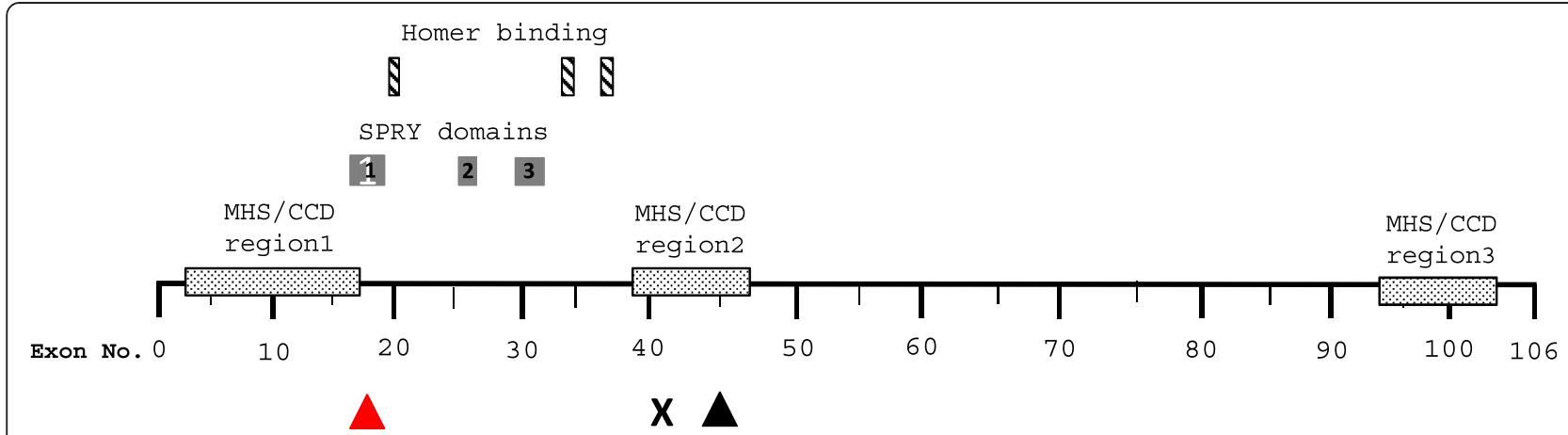

Figure 4 Location of nonsense mutation in MPS001 (X) and in frame deletions in Family MPS002 (red triangle) and Family MPS003 (black triangle) in relation to exon structure and RYR1 protein domains. Malignant Hyperthermia/central core disease mutation hot spots shown as stippled boxes, SPRY2 interacting domains 1,2 \& 3 as grey boxfibres, Homer binding motifs as hatched boxes.

(Figure 3E-H). Labelling for active caspase 3 excluded apoptotic loss of muscle fibres (Figure 3I). Ultrastructural analysis demonstrated hypotrophy with profound myofibrillar disarray and Z-disc loss (Figure 3K,L). Analysis of the spinal cord showed no loss of motor neurons or other pathological signs in the anterior horns and most proximal segments of the motor nerves, supporting the myogenic nature of the histological and ultrastructural findings.

\section{Family MPS003}

The muscle appearance was striking but non-specific. There was variation in myofibre size with larger hyalinised rounded fibres and smaller rounded atrophic fibres with an apparent increase in fibrous tissue. Fast and slow myosin heavy chains were co-expressed in a proportion of foetal myofibres. Occasional scattered chronic inflammatory cells were confirmed on CD3 and CD20 staining. Additional CD45 staining was interpreted as non-specific. Gomori trichrome staining was negative for nemaline rods and ragged red fibres. The couple had a previous intrauterine death at 23 weeks of gestation of a similarly affected male foetus (Figure 3M-O).

\section{Discussion}

After undertaking genetic linkage studies in a consanguineous family with recurrent FADS/LMPS we mapped a large candidate autozygous region to chromosome 19 and then proceeded to identify a homozygous nonsense mutation in the ryanodine receptor 1 (RYR1) gene. RYR1 encodes the largest known ion channel and the 2.3 MDa homotetrameric ryanodine receptor 1 structure is formed from the $565 \mathrm{kDa}$ RYR1 component proteins. Though the large size ( $15.3 \mathrm{~kb}$ coding sequence in 106 exons) of the RYR1 gene makes mutation analysis challenging, RYR1 mutations have previously been described in a variety of human disease phenotypes. Thus initially RYR1 mutations were described in individuals susceptible to malignant hyperthermia (MHS) [17,18]. RYR1 mutations associated with MHS are typically heterozygous missense substitutions [19-21]. Subsequently RYR1 mutations were described in the context of a variety of histological subtypes of congenital myopathies including central core disease, minicore/centronuclear myopathy with external ophthalmoplegia, centronuclear myopathy and congenital fibre-type disproportion [22-24]. Both dominant and recessive forms of $R Y R 1$-related congenital myopathies have been described and genotype-phenotype correlations have provided insights into likely clinical-functional relationships. Thus, in a large cohort of RYR1-associated myopathies, dominant mutations tended to be associated with milder phenotypes whereas recessively inherited cases had an earlier onset and a more severe course [25-27]. In addition, though the age at presentation of recessive $R Y R 1$ myopathies is variable, in a recent series all presented before age 10 years. Whereas RYR1 mutations in dominantly inherited disease tend to cluster in specific protein domains e.g. C-terminal region (amino acids $4,550-4,940)$ in central core disease and N-terminal regions (amino acids 35-614 and 2,163-2,458) with MHS, recessively inherited mutations are widely distributed throughout the protein [25,27]. Typically the mutations found in patients with recessively inherited RYR1-myopathies are a combination of null mutation with a missense mutation, though two missense mutations can occur. Thus in a series of 118 patients with RYR1-related recessively inherited myopathies from four recent reports [25,27-30], $61.5 \%$ of the cases had a truncating/in frame deletion/splice site mutation in combination with a missense mutation and $38.1 \%$ harboured two missense mutations. It is therefore striking that we identified a homozygous null mutation in affected individuals from Family MPS001. This observation is consistent with (a) our previous observation for RAPSN that homozygosity for a null mutation can cause FADS/LMPS other 
mutation combinations with only a single null mutation can cause a milder phenotype, (b) that mice homozygous for a Ryr1 mutation die in the perinatal period with gross abnormalities of skeletal muscle [31] and (c) a history of foetal akinesia may be found with early onset autosomal recessive RYR1-related congenital myopathies [25]. In addition, Romero et al. [32] reported seven foetuses/infants from six unrelated families affected by central core disease in whom there was a history of foetal akinesia [33]. Four cases from three families were found to harbour RYR1 mutations: three cases (from two families) were compound heterozygotes for RYR1 missense mutations and in one case only a heterozygous missense mutation was detected. Three of the four cases presented at birth and though in one case the foetus died at 32 weeks gestation (following termination of pregnancy after a previously affected sibling). Thus the phenotype in these cases was less severe than we observed and our findings demonstrate that the association between RYR1 mutations and foetal akinesia extends to severe early onset lethal FADS and that histopathological evidence of central core disease is not a prerequisite for molecular investigation of $R Y R 1$ in foetal akinesia.

The overall frequency of $R Y R 1$-related disease in our FADS/LMPS/EVMPS cohort was 4.5\% (3/66; 95\% CI: 0 to $9.5 \%)$ and $8.3 \%(3 / 36 ; 95 \%$ CI 0 to $19.5 \%)$ in our FADS/LMPS cohort. The case for pathogenicity of the two in frame deletions is supported by their absence from large repositories of genetic variation in control individuals, segregation with disease within the relevant families and evolutionary conservation of the mutated/ deleted amino acid residues. RYR1 is a key component of the excitation-coupling process in skeletal muscle such that opening of RYR1 channels result in release of $\mathrm{Ca}^{2+}$ from the sarcoplasmic reticulum and initiation of muscle contraction. The novel in-frame deletion of 27 nucleotides (c.2097_2123del p.(Glu699_Gly707del)) detected in Family MPS002 is predicted to result in a missense substitution (p.E699N) followed by a deletion of 9 amino acids (GWGGNGVGD) within the SPRY2 predicted proteinprotein interaction motif [32] (highlighted in Figure 4). Previously missense substitutions within or adjacent to this deletion (c.2113G > C; p.Gly705Arg and p.Asp708Asn) have been reported in recessively inherited myopathies [25]. The exon 45 in-frame deletion (c.7043delGAG) identified in Family MPS003 was predicted to result in loss of a glutamic acid residue at codon 2347. A missense mutation at a nearby residue (p.Arg2355Trp) has been reported in both dominantly and recessively inherited myopathies [20] and it is interesting that this deletion was previously described in the heterozygous state, in two unrelated families with malignant hyperthermia [34]. p.Glu2347 is contained within the MHS/CCD mutation hotspot in N-terminal region 2 (stippled box Figure 4),
[16]. Though no history of malignant hyperthermia syndrome was reported in Family MPS003, incomplete penetrance is well recognised in malignant hyperthermia and mutation carriers may not have been exposed to trigger events.

Though our findings establish recessive RYR1 mutations as a cause FADS/LMPS, further work is required to fully establish the frequency of RYR1 mutations in FADS/LMPS cohorts and to address how novel missense or in-frame deletions/insertions might be reliably interpreted in a clinical diagnostic setting. In a recent review of congenital myopathies treated at a single referral centre, a genetic diagnosis was established in two-thirds of cases and almost $60 \%$ of those with a genetic diagnosis had a RYR1-related myopathy [27]. Our findings suggest that RYR1-related neuromuscular disease may be a significant cause of FADS/LMPS. Though recessively inherited RYR1-related myopathies have been associated with certain histopathological subtypes such as minicore, centronuclear and congenital fibre-type disproportion myopathies, RYR1 mutations may be associated with other histological subtypes or only nonspecific myopathic features [27]. Extrapolating from these observations, we suggest that, in cases of FADS/LMPS, RYR1 mutation should be performed as part of a multigene diagnostic strategy (e.g. by second generation sequencing analysis) rather than being specifically targeted to cases with histopathological features that are considered characteristic of a RYR1-associated myopathy. The identification of RYR1 mutations as a cause of familial LMPS/ foetal akinesia enables accurate reproductive risk prediction and reproductive options including prenatal diagnosis and pre-implantation diagnosis but also might lead to the identification of relatives at risk of malignant hyperthermia.

\section{Abbreviations}

RYR1: Ryanodine Receptor 1 (skeletal); MPS: Multiple Pterygium Syndrome; FADS: Foetal Akinesia Deformation Sequence; LMPS: Lethal Multiple Pterygium Syndrome; EVMPS: Escobar-type Multiple Pterygium Syndrome AChR: Acetylcholine Receptor; CHRNG: Cholinergic Receptor Nicotinic Gamma subunit; CHRNA1: Cholinergic Receptor Nicotinic Alpha1 subunit: CHRND: Cholinergic Receptor Nicotinic Delta subunit; RAPSN: Receptor Associated Protein of the Synapse; DOK7: Docking Protein 7;

CMS: Congenital Myesthenic Syndrome; MHS: Malignant Hyperthermia Susceptibility; CCD: Central Core Disease.

\section{Competing interests}

The authors declare that they have no competing interests.

\section{Authors' information}

Arthur B McKie, Atif Al-Saedi, Julie Vogt, Kyra E Stuurman joint first authorship.

\section{Acknowledgements}

We thank NewLife and the NIHR Cambridge Biomedical Research Centre for financial support, and we thank the families and referring clinicians for their help with this research.

\section{Author details}

${ }^{1}$ Department of Medical Genetics, University of Cambridge and NIHR Cambridge Biomedical Research Centre, Cambridge Biomedical Campus, 
Cambridge CB2 0QQ, UK. ${ }^{2}$ Centre for Rare Diseases and Personalised Medicine, University of Birmingham, Edgbaston, Birmingham B15 2TT, UK. ${ }^{3}$ West Midlands Regional Genetics Service, Birmingham Women's Hospital, Birmingham B15 2TG, UK. ${ }^{4}$ Department of Clinical Genetics, VU University Medical Center, Amsterdam, The Netherlands. ${ }^{5}$ Department of Clinical Genetics, Addenbrooke's Treatment Centre, Cambridge University Hospitals NHS Foundation Trust, Hills Road, Cambridge CB2 0QQ, UK. ${ }^{6}$ Department of Medical Genetics, University Medical Center Utrecht, University of Utrecht, Utrecht, The Netherlands. 'Department of Pathology, VU University Medical Center, Amsterdam, the Netherlands. ${ }^{8}$ Department of Pathology, University Medical Center Utrecht, Utrecht, The Netherlands. ${ }^{9}$ Department of Medical Genetics, School of Clinical Medicine, University of Cambridge,

Addenbrooke's Treatment Centre, Cambridge Biomedical Campus, Box 238, Cambridge CB2 OQQ, United Kingdom.

\section{Received: 13 August 2014 Accepted: 6 October 2014} Published online: 05 December 2014

\section{References}

1. Hall JG, Reed SD, Rosenbaum KN, Gershanik J, Chen H, KM W, Limb (1982) pterygium syndromes: a review and report of eleven patients. Am J Hum Genet 12:377-379

2. McKeown CM, Harris R (1988) An autosomal dominant multiple pterygium syndrome. J Med Genet 25:96-103

3. Prontera P, Sensi A, Merlo L, Garani G, Cocchi G, E C (2006) Familial occurrence of multiple pterygium syndrome: expression in a heterozygote of the recessive form or variability of the dominant form? Am J Med Genet A 140:2227-2230

4. Tolmie JL, Patrick A, JR Y (1987) A lethal multiple pterygium syndrome with apparent X-linked recessive inheritance. Am J Med Genet A 27:913-919

5. Cox PM, Brueton LA, Bjelogrlic P, Pomroy P, CA S (2003) Diversity of neuromuscular pathology in lethal multiple pterygium syndrome. Pediatr Dev Pathol 6:59-68

6. Hoffmann K, Muller JS, Stricker S, Megarbane A, Rajab A, Lindner TH, Cohen M, Chouery E, Adaimy L, Ghanem I, Delague V, Boltshauser E, Talim B, Horvath R, Robinson PN, Lochmuller H, Hubner C, S M (2006) Escobar syndrome is a prenatal myasthenia caused by disruption of the acetylcholine receptor fetal gamma subunit. Am J Hum Genet 79:303-312

7. Morgan NV, Brueton LA, Cox P, greally MT, Tolmie J, Pasha S, Aligianis IA, van Bokhoven $\mathrm{H}$, Marton T, Al-Gazali L, Morton JEV, Oley C, Johnson CA, Trembath RC, Brunner HG ERM (2006) Mutations in the embryonal subunit of the acetylcholine receptor (CHRNG) cause lethal and Escobar variants of multiple pterygium syndrome. Am J Hum Genet 79:390-395

8. Michalk A, Stricker S, Becker J, Rupps R, Pantzar T, Miertus J, Botta G, Naretto VG, Janetzki C, Yaqoob N, Ott CE, Seelow D, Wieczorek D, Fiebig B, Wirth B, Hoopmann M, Walther M, Körber F, Blankenburg M, Mundlos S, Heller R, K $H$ (2008) Acetylcholine receptor pathway mutations explain the fetal akinesia deformation sequence. Am J Hum Genet 82:464-476

9. Vogt J, Harrison BJ, Spearman H, Cossins J, Vermeer S, ten Cate LN, Morgan NV, Beeson D, ER M (2008) Mutation analysis of CHRNA1, CHRNB1, CHRND, and RAPSN genes in multiple pterygium syndrome/fetal akinesia patients. American Journal of Human Genetics 82:222-227

10. Vogt J, Morgan NV, Marton T, Maxwell S, Harrison BJ, Beeson D, Maher ER (2008) Germline mutation in DOK7 associated with fetal akinesia deformation sequence. J Med Genet 46:338-340

11. Beeson D, Hantai D, Lochmuller H, Engel AG (2004) 126 International Workshop: congenital myasthenic syndromes. Neuromuscul Disord 15:498-515

12. Brownlow S, Webster R, Croxen R, Brydson M, Neville B, Lin JP, Vincent A, Newsom-Davis J, D B (2001) Acetylcholine receptor $\delta$ subunit mutations underlie a fast-channel myasthenic syndrome and arthrogryposis multiplex congenita. J Clin Invest 108:125-130

13. Burke G, Cossins J, Maxwell S, Owens G, Vincent A, Robb S, Nicolle M, Hilton-Jones D, Newsom-Davis J, Palace J, D B (2003) Rapsyn mutations in hereditary myasthenia: distinct early- and late-onset phenotypes. Neurology 23:826-828

14. World Medical Association Declaration of Helsinki (1964) Recommendations guiding physicians in biomedical research involving human subjects. BMJ 313:1448

15. Bancroft JD, Stevens A (eds) (2008) Theory and practice of histological techniques, 4th edn. Churchill Livingstone Elsevier, Churchill Livingstone Edinburgh 1986, ISBN: 978-0-443-10279-0
16. Takeshima $H$, lino M, Takekura H, Nishi M, Kuno J, Minowa O, Takano H, T N (1994) Excitation-contraction uncoupling and muscular degeneration in mice lacking functional skeletal muscle ryanodine-receptor gene. Nature 369:556-559

17. Fujii J, Otsu K, Zorzato F, de Leon S, Khanna VK, Weiler JE, O'Brien PJ, DH M (1991) Identification of a mutation in porcine ryanodine receptor associated with malignant hyperthermia. Science 253:448-451

18. Gillard EF, Otsu K, Fujii J, Khanna VK, de Leon S, Derdemezi J, Britt BA, Duff CL, Worton RG, DH M (1991) A substitution of cysteine for arginine 614 in the ryanodine receptor is potentially causative of human malignant hyperthermia. Genomics 11:751-755

19. Brandom BW, Bina S, Wong CA, Wallace T, Visolu M, Isackson PJ, Vladutiu GD, Sambuughin N, Muldoon SM (2013) Ryanodine receptor type1 gene variants in the malignant hyperthermia-susceptible population of the United States. Anesth Analg 116:1078-1086

20. Kim JH, Jarvik GP, Browning BL, Rajagopalan R, Gordon AS, Rieder MJ, Robertson PD, Nickerson DA, Fisher NA (2013) Exome sequencing reveals novel rare variants in the Ryanodine receptor and calcium channel genes in malignant hyperthermia families. Anesthesiology 119:1054-1065

21. Broman M, Heinecke K, Islander G, Schuster F, Glahn K, Bodelsson M, Treves S (2011) Screening of the Ryanodine receptor type1 gene for malignant hyperthermia causative mutations by high resolution melt curve analysis. Anesth Analg 113:1120-1128

22. Clarke NF, Waddell LB, Cooper ST, Perry M, Smith RL, Kornberg AJ, Muntoni F, Lillis S, Straub V, Bushby K, Guglieri M, King MD, Farrell MA, Marty I, Lunardi J, Monnier N, KN N (2010) Recessive mutations in RYR1 are a common cause of congenital fiber type disproportion. Human Mutation 31: E1544-E1550

23. Treves S, Jungbluth H, Muntoni F, F Z (2008) Congenital muscle disorders with cores: the ryanodine receptor calcium channel paradigm. Current Opinions in Pharmacology 8:319-326

24. Wilmshurst JM, Lillis S, Zhou H, Pillay K, Henderson H, Kress W, Müller CR, Ndondo A, Cloke V, Cullup T, Bertini E, Boennemann C, Straub V, Quinlivan R, Dowling JJ, Al-Sarraj S, Treves S, Abbs S, Manzur AY, Sewry CA, Muntoni F, H J (2010) RYR1 mutations are a common cause of congenital myopathies with central nuclei. Annals of Neurology 68:717-726

25. Klein A, Lillis S, Munteanu I, Scoto M, Zhou H, Quinlivan R, Straub V, Manzur AY, Roper $H$, Jeannet PY, Rakowicz W, Jones DH, Jensen UB, Wraige $E$, Trump N, Schara U, Lochmuller H, Sarkozy A, Kingston H, Norwood F, Damian M, Kirschner J, Longman C, Roberts M, Auer-Grumbach M, Hughes I, Bushby K, Sewry C, Robb S, Abbs S et al (2012) Clinical and genetic findings in a large cohort of patients with ryanodine receptor 1 gene-associated myopathies. Human Mutation 33:981-988

26. Hwang JH, Zorzato F, Clarke NF, Treves S (2012) Mapping domains and mutations on the skeletal muscle ryanodine receptor channel. Trends Mol Med 18(11):644-657

27. Maggi L, Scoto M, Cirak S, Robb SA, Klein A, Lillis S, Cullup T, Feng L, Manzur AY, Sewry CA, Abbs S, Jungbluth H, F M (2013) Congenital myopathies-clinical features and frequency of individual subtypes diagnosed over a 5-year period in the United Kingdom. Neuromuscul Disord 23:195-205

28. Amburgey K, Bailey A, Hwang J, Bonnemann C, Medna L, Matthews K, Collins J, Daube J, Wellman G, Callaghan B, Clarke N, Dowling J (2013) Genotype-phenotype correlations in recessive RYR1-related myopathies. Orphanet J Rare Dis 8:1-12

29. Bharucha-Goebel DX, Medne L Zukosky K, Dastgir J, Sheih PB, Winder T, Tennekoon G, Finkel RS, Dowling JJ, Monnier N, Bonnemann CG (2013) Severe congenital RYR1-associated myopathy: the expanding clinicopathologic and genetic spectrum. Neurology 80:1584-1589

30. Zhou H, Jungbluth $H$, Sewry CA, Feng L, Bertini E, Bushby K, Straub V, Roper $H$, Rose MR, Brockington M, Kinali M, Manzur A, Robb S, Appleton R, Messina S, D’Amico A, Quinlivan R, Swash M, Müller CR, Brown S, Treves S, F M (2007) Centronuclear myopathy due to a de novo dominant mutation in the skeletal muscle ryanodine receptor (RYR1) gene. Neuromuscul Disord 17:338-345

31. Otsu K, Khanna VK, Archibald AL, DH M (1991) Cosegregation of porcine malignant hyperthermia and a probable causal mutation in the skeletal muscle ryanodine receptor gene in backcross families. Genomics 11:744-750

32. Perálvarez-Marín A, Tae H, Board PG, Casarotto MG, Dulhunty AF, M S (2011) 3D Mapping of the SPRY2 domain of ryanodine receptor 1 by single-particle cryo-EM. PLoS One 6:e25813 
33. Romero NB, Monnier N, Viollet L, Cortey A, Chevallay M, Leroy JP, Lunardi J, Fardeau M (2003) Dominant and recessive central core disease associated with RYR1 mutations and fetal akinesia. Brain 126:2341-2349

34. Sambuughin N, McWilliams S, de Bantel A, Sivakumar K, TE N (2001)

Single-amino-acid deletion in the RYR1 gene, associated with malignant hyperthermia susceptibility and unusual contraction phenotype. Am J Hum Genet 69:204-208

doi:10.1186/s40478-014-0148-0

Cite this article as: McKie et al: Germline mutations in RYR1 are

associated with foetal akinesia deformation sequence/lethal multiple

pterygium syndrome. Acta Neuropathologica Communications 2014 2:148.

\section{Submit your next manuscript to BioMed Central and take full advantage of:}

- Convenient online submission

- Thorough peer review

- No space constraints or color figure charges

- Immediate publication on acceptance

- Inclusion in PubMed, CAS, Scopus and Google Scholar

- Research which is freely available for redistribution 